\title{
NOTE ON THE PROBLEM OF THE DETERMINATION OF A PLANET'S ORBIT FROM THREE OBSERVATIONS.
}

[From the Monthly Notices of the Royal Astronomical Society, vol. xxix. (1868-1869), pp. 257-259.]

THE principle of the solution given in the Theoria Motus may be explained very simply as follows:

Consider three successive positions of $C, C^{\prime}, C^{\prime \prime}$, of a planet revolving about the focus $S$; let $n, n^{\prime}, n^{\prime \prime}$, denote the doubles of the triangular areas $C^{\prime} S C^{\prime \prime}, C S C^{\prime}$, and $C S C^{\prime \prime}$ respectively (viz. the triangular area means the area of the triangle included between the two radius vectors and the chord joining their extremities), $r^{\prime}$ the radius

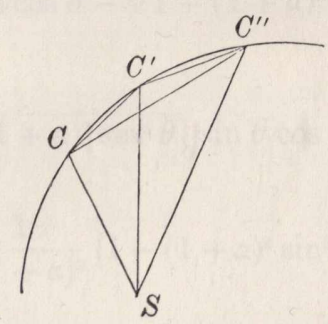

vector $S C^{\prime} ; \theta^{\prime \prime}, \theta$, the times of describing the arcs $C C^{\prime}$ and $C^{\prime} C^{\prime \prime}$ respectively, the units of time and distance being such that the time is equal to the double area divided by the square root of the half latus rectum $\left(t=2 \pi a^{\frac{3}{2}}\right.$ for the Period in a circular or elliptic orbit).

Then writing

$$
P=\frac{n^{\prime \prime}}{n}, \quad Q=2\left(\frac{n+n^{\prime \prime}}{n^{\prime}}-1\right) r^{\prime 3}
$$


(observe that $n+n^{\prime \prime}-n^{\prime}$ is =twice the triangle $C C^{\prime \prime} C^{\prime \prime}$ ), for neighbouring positions of the planet, the values of $P$ and $Q$ are approximately $=\frac{\theta^{\prime \prime}}{\theta}$ and $\theta \theta^{\prime \prime}$ respectively: the solution consists in the determination of an orbit for which $P$ and $Q$ have these approximate values; then, by means of such approximate orbit, the values of $P$ and $Q$ are more accurately determined, and by means of these new values of $P$ and $Q$, a new determination is effected of the orbit: and so on, to the requisite accuracy of approximation.

The foregoing approximate values of $P$ and $Q$ respectively are deduced from the accurate values

$$
P=\frac{\theta^{\prime \prime} \eta}{\theta \eta^{\prime \prime}}, \quad Q=\frac{\theta \theta^{\prime \prime}}{\eta \eta^{\prime \prime}} r^{\prime} r^{\prime \prime} \frac{1}{\cos f \cos f^{\prime} \cos f^{\prime \prime}} ;
$$

where $r, r^{\prime}, r^{\prime \prime}$ are the radius vectors $S C, S C^{\prime}, S C^{\prime \prime} ; 2 f, 2 f^{\prime}, 2 f^{\prime \prime}$ are the angular distances $C^{\prime} S C^{\prime \prime}, C S C^{\prime \prime}, C S C^{\prime}\left(f^{\prime}=f+f^{\prime \prime}\right)$ and $\eta, \eta^{\prime}, \eta^{\prime \prime}$ are the ratios of the sectorial areas $C^{\prime} S C^{\prime \prime}, C S C^{\prime \prime}, C^{\prime} S C^{\prime \prime}$, to the triangular areas represented by the same letters respectively: the doubles of the sectorial areas are thus $n \eta, n^{\prime} \eta^{\prime}$, and $n^{\prime \prime} \eta^{\prime \prime}$, and if the half latus rectum be denoted by $p$, then we have

$$
\sqrt{p}=\frac{n \eta}{\theta}=\frac{n^{\prime} \eta^{\prime}}{\theta^{\prime}}=\frac{n^{\prime \prime} \eta^{\prime \prime}}{\theta^{\prime \prime}}
$$

and it thus at once appears that the accurate value of $P$ is $=\frac{\theta^{\prime \prime} \eta}{\theta \eta^{\prime \prime}}$, as above. To obtain the expression for $Q$, taking $\phi, \phi^{\prime}, \phi^{\prime \prime}$ for the true anomalies (and, for greater symmetry, writing for the moment $\nu,-\nu^{\prime}, \nu^{\prime \prime}, g,-g^{\prime}, g^{\prime \prime}$ in place of $n, n^{\prime}, n^{\prime \prime}, f, f^{\prime}, f^{\prime \prime}$ respectively), we have

whence identically

$$
\begin{aligned}
& r=\frac{p}{1+e \cos \phi}, 2 g=\phi^{\prime \prime}-\phi^{\prime}, \\
& r^{\prime}=\frac{p}{1+e \cos \phi^{\prime}}, 2 g^{\prime}=\phi-\phi^{\prime \prime}, \\
& r^{\prime \prime}=\frac{p}{1+e \cos \phi^{\prime \prime}}, 2 g^{\prime \prime}=\phi^{\prime}-\phi, \\
&\left(g+g^{\prime}+g^{\prime \prime}=0\right) ;
\end{aligned}
$$

or writing

this is

$$
\frac{\sin 2 g}{r}+\frac{\sin 2 g^{\prime}}{r^{\prime}}+\frac{\sin 2 g^{\prime \prime}}{r^{\prime \prime}}=-\frac{4 \sin g \sin g^{\prime} \sin g^{\prime \prime}}{p}
$$

$$
\nu=r^{\prime} r^{\prime \prime} \sin 2 g, \quad \nu^{\prime}=r^{\prime \prime} r \sin 2 g^{\prime}, \quad \nu^{\prime \prime}=r r^{\prime} \sin 2 g^{\prime \prime},
$$

$$
\begin{aligned}
\nu+\nu^{\prime}+\nu^{\prime \prime} & =-\frac{4 r r^{\prime} r^{\prime \prime} \sin g \sin g^{\prime} \sin g^{\prime \prime}}{p}, \\
& =-\frac{\left(r r^{\prime} r^{\prime \prime}\right)^{2} \sin 2 g \sin 2 g^{\prime} \sin 2 g^{\prime \prime}}{2 p r r^{\prime} r^{\prime \prime} \cos g \cos g^{\prime} \cos g^{\prime \prime}} \\
& =-\frac{\nu \nu^{\prime} \nu^{\prime \prime}}{2 p r r^{\prime} r^{\prime \prime} \cos g \cos g^{\prime} \cos g^{\prime \prime}} .
\end{aligned}
$$

c. VII. 
This is, in fact,

$$
n-n^{\prime}+n^{\prime \prime}=\frac{n n^{\prime} n^{\prime \prime}}{2 p r r^{\prime} r^{\prime \prime} \cos f^{\prime} \cos f^{\prime} \cos f^{\prime \prime}},
$$

or since

$$
\frac{n n^{\prime \prime}}{p}=\frac{\theta \theta^{\prime}}{\eta \eta^{\prime \prime}}
$$

it is

$$
2\left(\frac{n+n^{\prime \prime}}{n^{\prime}}-1\right)=\frac{\theta \theta^{\prime \prime}}{\eta \eta^{\prime \prime} r r^{\prime} r^{\prime \prime} \cos f \cos f^{\prime} \cos f^{\prime \prime}},
$$

viz. multiplying by $r^{\prime 3}$, it is

$$
Q=\begin{aligned}
& \theta \theta^{\prime \prime} \\
& \eta \eta^{\prime \prime} \\
& r r^{\prime \prime}
\end{aligned} \frac{1}{\cos f \cos f^{\prime} \cos f^{\prime \prime}}
$$

the above-mentioned value of $Q$. 